\title{
Reducing the Time \\ to Determine Content Radioactive Aerosols \\ in the Air of Industrial Premises
}

\author{
Tatiana A. Kulagina* and Sergey A. Ivanov \\ Siberian Federal University \\ Krasnoyarsk, Russian Federation
}

Received 17.11.2020, received in revised form 20.11.2020, accepted 29.11.2020

\begin{abstract}
The possibility of improving the methodology for measuring the content of radioactive aerosols in indoor air is considered, in terms of time reducing for determining the required parameters without serious changes in the design of the equipment applied and without reducing the safety of the radiation object itself. It is advisable to design a computational model that compares dynamics of the $\alpha$-decay of a sample with a certain calibration curve of decay obtained for a given region. In this case, the time for determining the amount of technogenic radioactive aerosols in indoor air is reduced by 72 times.
\end{abstract}

Keywords: radiation safety, radioactive aerosols emanation half-life periods of radionuclides, $\alpha$-activity background samples aerosols.

Citation: Kulagina T.A., Ivanov S.A. Reducing the time to determine content radioactive aerosols in the air of industrial premises, J. Sib. Fed. Univ. Eng. \& Technol., 2020, 13(8), 965-976. DOI: 10.17516/1999-494X-0276

\section{О сокращении времени определения \\ содержания радиоактивных аэрозолей \\ в воздухе производственных помещений}

\author{
Т.А. Кулагина, С.А. Иванов \\ Сибирский федеральный университет \\ Российская Федераиия, Красноярск
}

Аннотация. Рассмотрена возможность усовершенствования методики измерения содержания радиоактивных аэрозолей в воздухе помещений, в части сокращения времени определения

(C) Siberian Federal University. All rights reserved

This work is licensed under a Creative Commons Attribution-Non Commercial 4.0 International License (CC BY-NC 4.0).

* Corresponding author E-mail address: tak.sfu@gmail.com 
требуемых параметров без серьезных изменений в конструкции используемого оборудования и без снижения безопасности самого радиационного объекта. Целесообразно создание расчетной модели, сравнивающей динамику $\alpha$-распада пробы с некой калибровочной кривой распада, полученной для данного региона. В этом случае сокращается время определения количества техногенных радиоактивных аэрозолей в воздухе помещений в 72 раза.

Ключевые слова: радиационная безопасность, радиоактивные аэрозоли, эманация, периоды полураспада радионуклидов, $\alpha$-активность фоновых проб аэрозолей.

Цитирование: Кулагина, Т.А. О сокращении времени определения содержания радиоактивных аэрозолей в воздухе производственных помещений / Т.А. Кулагина, С.А. Иванов // Журн. Сиб. федер. ун-та. Техника и технологии, 2020. 13(8). C. 965-976. DOI: 10.17516/1999-494X-0276

\section{Введение}

Существующая в нашей стране система обращения с радиоактивными отходами (РАO) сформировалась в процессе развития в СССР ядерных технологий и лежит в русле международных конвенций $[1,2]$, признанных Российской Федерацией, но имеет ряд характерных особенностей, которые отличают ее от подобных систем других стран [3-9]. Обеспечение ядерной и радиационной безопасности в России регулируется собственной правовой и организационной системой [9-13]. Технологии обращения с радиоактивными отходами и трудно растворимыми осадками рассмотрены в [14-20].

В результате вывода из эксплуатации атомных подводных лодок (АПЛ) происходит накопление их в пунктах базирования, в основном находящихся на арктическом побережье. Хранение на плаву с невыгруженными активными зонами реакторных установок создает источник радиационной опасности глобального масштаба [8]. В настоящее время организованы и функционируют промышленные предприятия по хранению и переработке отработавшего ядерного топлива АПЛ. Одним из важнейших аспектов надежного функционирования таких предприятий является радиационная безопасность. Радиационная безопасность есть состояние защищенности настоящего и будущего поколений людей от вредного для их здоровья воздействия ионизирующего излучения [12].

Для обеспечения радиационной безопасности при нормальной эксплуатации источников излучения необходимо руководствоваться следующими основными принципами:

- непревышение допустимых пределов индивидуальных доз облучения граждан от всех источников излучения (принцип нормирования);

- запрещение всех видов деятельности по использованию источников излучения, при которых полученная для человека и общества польза не превышает риск возможного вреда, причиненного дополнительным облучением (принцип обоснования);

- поддержание на возможно низком и достижимом уровне с учетом экономических и социальных факторов индивидуальных доз облучения и числа облучаемых лиц при использовании любого источника излучения (принцип оптимизации).

Для обоснования расходов на радиационную защиту при реализации принципа оптимизации принимается, что облучение в коллективной эффективной дозе в 1 чел·Зв приводит к потенциальному ущербу, равному потере примерно 1 чел·года жизни населения. Величина денежного эквивалента потери 1 чел·года жизни устанавливается отдельными документами федерального уровня в размере не менее одного годового душевого национального дохода.

$$
-966-
$$


Для наиболее полной оценки вреда, который может быть нанесен здоровью в результате облучения в малых дозах, определяется ущерб, количественно учитывающий эффекты облучения как отдельных органов и тканей тела, отличающихся радиочувствительностью к ионизирующему излучению, так и всего организма в целом. В соответствии с общепринятой в мире линейной беспороговой теорией зависимости риска стохастических эффектов от дозы величина риска пропорциональна дозе излучения и связана с дозой через линейные коэффициенты радиационного риска [13], приведенные в табл. 1.

Усредненная величина коэффициента риска, используемая для установления пределов доз персонала и населения, принята равной 0,05 Зв $^{-1}$.

В условиях нормальной эксплуатации источников ионизирующего излучения пределы доз облучения в течение года устанавливают, исходя из следующих значений индивидуального пожизненного риска:

- для персонала $-1,0 \times 10^{-3}$;

- для населения $-5,0 \times 10^{-5}$.

Уровень пренебрежимо малого риска равен 10. При обосновании защиты от источников потенциального облучения в течение года принимаются следующие граничные значения обобщенного риска (произведение вероятности события, приводящего к облучению, и вероятности смерти, связанной с облучением):

- персонал - 2,0×10-4 год $^{-1}$;

- население $-1,0 \times 10^{-5}$, год ${ }^{-1}$.

Радиоактивными аэрозолями называют любые взвешенные частицы, находящиеся в воздухе и имеющие естественную (продукты распада урана, тория или радия) или искусственную (продукты деления урана, активационные радионуклиды и др.) радиоактивность. В зависимости от агрегатного состояния различают аэрозоли с твердой дисперсной фазой (пыль различных радиоактивных веществ, дым) и аэрозоли с жидкой дисперсной фазой (пар, туман или аэрозоли конденсации).

Естественными носителями радиоактивности атмосферного воздуха являются эманации (радон, торон, актинион) и их дочерние продукты (радионуклиды полония, висмута, свинца и талия), которые могут осесть на взвешенных в воздухе частицах, тогда как твердые продукты распада эманаций в результате объемной конденсации сами могут образовывать аэрозоли различной дисперсности.

Радиоактивные аэрозоли образуются в процессах производства и обработки радиоактивных материалов (дробление, механическая обработка, выпаривание, кипячение и т.п.), при

Таблица 1. Линейные коэффициенты радиационного риска

Table 1. Linear radiation risk factors

\begin{tabular}{|c|c|c|c|}
\hline $\begin{array}{c}\text { Облучаемая группа } \\
\text { населения }\end{array}$ & $\begin{array}{c}\text { Коэффициент риска } \\
\text { 3локачественных новообразований, } \\
\times 10^{-2} \text { Зв } \text { - }^{-1}\end{array}$ & $\begin{array}{c}\text { Коэффициент риска } \\
\text { наследственных эффектов, } \\
\times 10^{-2} \text { Bв }^{-1}\end{array}$ & $\begin{array}{c}\text { Сумма, } \\
\times 10^{-2} \text { 3 }^{-1}\end{array}$ \\
\hline Все население & 5,5 & 0,2 & 5,7 \\
\hline Взрослые & 4,1 & 0,1 & 4,2 \\
\hline
\end{tabular}


взрывах атомных и водородных бомб, при взаимодействии космического излучения с атмосферной пылью [21].

Важным источником радиоактивных аэрозолей и газов служат ядерные реакторы и ядерное топливо атомных электрических станций (АЭС). При нормальной работе реактора происходит активация тепловыми нейтронами аргона. При нарушении целостности защитной оболочки тепловыделяющих элементов (ТВЭЛов) продукты деления ядерной реакции могут попасть в теплоноситель первого контура. В этом случае появляется реальная опасность загрязнения воздуха радиоактивными газами $\mathrm{Xe}, \mathrm{Kr}$, летучими (I, Cs и др.) и нелетучими веществами (Sr, Ba, La, Zr, Ru, Cе и др.).

Большая часть продуктов деления, а также продуктов их радиоактивного распада, как $\beta$ - и $\gamma$-частицы, радиоактивна. Их периоды полураспада находятся в широких пределах от долей секунды до десятка лет и более. В результате активации продуктов коррозии оборудования первого контура АЭС в теплоносителе могут находиться радионуклиды $\mathrm{Cr}^{51}, \mathrm{Mn}^{54}, \mathrm{Co}^{58}, \mathrm{Fe}^{59}, \mathrm{Co}^{60}$, $\mathrm{Zn}^{65}, \mathrm{Zr}^{98}$ и др. Кроме них в воде образуются короткоживущие радионуклиды $\mathrm{N}^{16}, \mathrm{~F}^{18}, \mathrm{Na}^{24}, \mathrm{~K}^{42}$ и другие, которые не представляют опасности при выходе в окружающую среду. То же можно сказать о долгоживущем тритии, который в небольшом количестве образуется вследствие активации дейтерия и ядерной реакции, происходящей на боре (борная кислота применяется для регулирования мощности реактора). Теплоноситель - охлаждающая вода с радионуклидами непрерывно направляется на специальную водоочистку с ионообменными фильтрами, которые очищают воду от радионуклидов, за исключением радиоактивных благородных газов (РБГ) и трития в форме тритиевой воды НТО [22].

Размер аэрозольных частиц в воздушной среде может находиться в различных пределах от долей до нескольких десятков микрометров. Распределение этих частиц по размерам, массе, а следовательно, и по активности подчиняется логарифмическому нормальному закону. В этом случае дисперсность аэрозольных частиц можно характеризовать медианным диаметром $d$.

Целью данной работы является определение возможности усовершенствования методики измерения содержания радиоактивных аэрозолей в воздухе, в части сокращения времени определения требуемых параметров без серьезных изменений в оборудовании.

\section{Свойства аэрозольных частиц в зависимости от их размера}

По дисперсности аэрозоли делятся на три группы:

1) крупнодисперсные (неустойчивые) частицы ( $d=10$ мкм и более) быстро оседают, поэтому находятся в воздухе недолго;

2) средней дисперсности (относительно устойчивые) частицы ( $d=1-10$ мкм) медленно оседают в неподвижном воздухе, поэтому находятся во взвешенном состоянии различное время в зависимости от размера частиц и их концентрации;

3) мелкодисперсные (устойчивые) частицы ( $d<1$ мкм) движутся подобно молекулам воздуха, т.е. подчиняются законам броуновского движения.

При столкновении между собой мелкодисперсные аэрозоли укрупняются (коагулируют) и оседают так же, как и аэрозоли средней дисперсности. Установлено, что коагуляция мелкодисперсных аэрозолей происходит тем быстрее, чем меньше размеры частиц и чем больше их концентрация. 
Несмотря на всевозможные защитные конструкции по задержанию радиоактивных газоаэрозолей (приточно-вытяжная вентиляция, боксы и т.п.) [17], какая-то часть их все же попадает в воздушную среду, а из нее в организм человека через органы дыхания. Согласно данным Международной комиссии по радиологической защите (МКРЗ), применяется следующее распределение вдыхаемых аэрозолей: осаждаются в верхних дыхательных путях 50 \%, осаждаются в легких $25 \%$, выдыхаются обратно в воздух $25 \%$. Установлено, что аэрозольные частицы средней дисперсности ( $d \geq 1$ мкм) эффективно задерживаются верхними дыхательными путями (в носоглотке откладываются 40-90\% частиц $d=1-10$ мкм; в трахеях и бронхах $-10 \%$ частиц $d=0,2-10$ мкм). Эти частицы могут попасть обратно в носоглотку, а затем в желудок и быть источником облучения желудочно-кишечного тракта. Некоторая часть из этих аэрозолей может всасываться в кровь [23].

\section{Методика определения радиоактивных аэрозолей}

Результаты многочисленных исследований показали, что значительную часть естественных радиоактивных изотопов можно по ряду тождественных генетических признаков сгруппировать в три радиоактивных семейства. Каждое такое семейство характеризуется наличием одного долгоживущего изотоп-родоначальника семейства, одного газообразного изотопа эманации, промежуточных радиоактивных продуктов распада и конечных стабильных изотопов с атомными весами 206, 207 и 208 соответственно. Родственной связи между остальными естественными радиоактивными изотопами, подобно связи между изотопами, отнесенными к упомянутым радиоактивным семействам, не установлено.

Естественные радиоактивные изотопы, как было выяснено в результате проведенных исследований объектов внешней среды, имеют в природе весьма широкое распространение и содержатся во всех оболочках Земли: атмосфере, гидросфере, литосфере и в биосфере. Однако в концентрированном виде естественные радиоактивные вещества встречаются довольно редко. Как правило, в различных сферах Земли они находятся в крайне рассеянном состоянии. В силу этого слабая радиоактивность стала неотъемлемым качеством воздуха, атмосферного и почвенного, воды морей, озер и рек, почвы, магматических, осадочных и метаморфических пород, а также ткани всех растительных и животных организмов. Кроме этого, естественные радиоактивные изотопы были обнаружены и в метеоритных телах, периодически падающих на нашу планету из космического пространства. Последнее обстоятельство имеет немаловажное значение, так как наряду с другими астрофизическими данными оно свидетельствует о том, что явление радиоактивности свойственно природе не только нашей планеты, но также имеет место в других образованиях Вселенной [24].

Многочисленными и часто очень трудоемкими исследованиями, производимыми после открытия явления радиоактивности, было установлено, что все радиоактивные изотопы, встречающиеся в природе и имеющие атомный номер от 81 до 92, входят в систему одного из радиоактивных семейств [25].

Интересующие нас радионуклиды встречаются в природе в виде изотопов - эманаций, их три: радон $\left({ }^{222} \mathrm{Rn}\right)$ продукт $\alpha$-распада ${ }^{226} \mathrm{Ra}$ (семейство $\left.{ }^{238} \mathrm{U}\right)$, период полураспада $T_{1 / 2}=3,825$ суток; торон $\left({ }^{220} \mathrm{Rn}\right)$ продукт $\alpha$-распада ${ }^{224} \mathrm{Ra}$ (семейство $\left.{ }^{232} \mathrm{Th}\right), T_{1 / 2}=51,5 \mathrm{c}$; актинон $\left({ }^{219} \mathrm{Rn}\right)$ продукт $\alpha$-распада ${ }^{223} \mathrm{Ra}$ (семейство $\left.{ }^{235} \mathrm{U}\right), T_{1 / 2}=3,9$ с [10].

$$
-969-
$$


Все эти эманации принадлежат к группе инертных газов, обладают $\alpha$-активностью и в основном других излучений не имеют. При распаде эманации образуется ряд короткоживущих твердых радиоактивных элементов, которые необходимо учитывать при определении радиационных параметров воздуха рабочей зоны. Причем количественные и качественные характеристики содержания в воздухе зависят от состава подстилающих горных пород, структуры почвы и характера атмосферных явлений в данном регионе. На рис. 1 представлены цепочки распада естественных эманаций трех основных радиоактивных семейств.

Поскольку периоды полураспада вышеуказанных естественных радионуклидов несравнимо менее продолжительны, чем у техногенных (к примеру, у плутония-239 он составляет 24000 лет), видится целесообразным сравнить динамику распада пробы аэрозолей с расчетной моделью распада смеси естественных радионуклидов. И с достаточной для оперативной оценки погрешностью спрогнозировать наличие и концентрацию техногенных радионуклидов, тем самым значительно сократив время определения аэрозолей техногенных радионуклидов в воздухе рабочей зоны.

Среди прочих одним из важнейших аспектов безопасности радиационных объектов является измерение содержания радиоактивных аэрозолей в воздухе рабочей зоны. В настоящее время количественные и качественные данные на предприятиях получают с использованием аттестованных методик определения таких параметров [26].

Суть методик определения радиоактивных аэрозолей в воздухе рабочей зоны в основном заключается в следующем:

- пробу аэрозолей путем протягивания воздушной среды с помощью аспиратора отбирают на фильтр из специальной ткани;

- фильтр выдерживают в течение 3 сут для распада естественных радионуклидов и затем измеряют на радиометре.

В действительности на промышленных предприятиях отсутствует возможность 3-суточного ожидания, поскольку часто возникает необходимость оперативно допустить персонал в помещение для выполнения срочных работ. И так как в воздухе помимо техногенных радионуклидов, которые, собственно, и необходимо определить, присутствуют естественные в схожих концентрациях и не отличающиеся по характеру излучения, осуществить оперативный допуск персонала при этом, выполнив все требования, не представляется возможным.

К примеру, контрольный уровень по содержанию аэрозолей плутония, являющегося альфа-излучающим радионуклидом, в воздухе рабочих помещений составляет 0,038 Бк/ $\mathrm{m}^{3}$, при этом $\alpha$-активность фильтра сразу после отбора может достигать за счет естественных радионуклидов 10 Бк/м².

1) ${ }^{222} \mathrm{Rn} \rightarrow 3,82$ сут $\rightarrow{ }^{218} \mathrm{Po} \rightarrow 3,11$ мин $\rightarrow{ }^{214} \mathrm{~Pb} \rightarrow 28,8$ мин $\rightarrow 19,9$ мин $\rightarrow 1,6 \mathrm{E}-4 \mathrm{c} \rightarrow$ $\rightarrow{ }^{210} \mathrm{~Pb} \rightarrow 21,8$ год $\rightarrow 5,01$ сут $\rightarrow{ }^{210} \mathrm{Po} \rightarrow 138,37$ сут $\rightarrow{ }^{206} \mathrm{~Pb}$;

2) ${ }^{220} \mathrm{Rn} \rightarrow 55 \mathrm{c} \rightarrow{ }^{216} \mathrm{Po} \rightarrow 0,145 \mathrm{c} \rightarrow{ }^{212} \mathrm{~Pb} \rightarrow 10,64 \mathrm{q} \rightarrow 60,55 \mathrm{мин} \rightarrow{ }^{212} \mathrm{Po} \rightarrow 2,9 \mathrm{E}-7 \mathrm{c} \rightarrow{ }^{208} \mathrm{~Pb}$;

3) $\quad{ }^{219} \mathrm{Rn} \rightarrow 3,96 \mathrm{c} \rightarrow{ }^{215} \mathrm{Po} \rightarrow 0,17 \mathrm{E}-2 \mathrm{c} \rightarrow{ }^{211} \mathrm{~Pb} \rightarrow 2,15$ мин $\rightarrow{ }^{207} \mathrm{Tl} \rightarrow 4,77$ мин $\rightarrow{ }^{207} \mathrm{~Pb}$.

Рис. 1. Цепочки распада естественных эманаций. Показаны исключительно $\alpha$-излучающие радионуклиды Fig. 1. Decay chains of natural emanations. Shown exclusively $\alpha$-emitting radionuclides

$$
-970-
$$




\section{Рекомендации по усовершенствованию методики}

В ходе эксперимента для отбора проб используют пробоотборное устройство ПУ-5. Пробы отбирают на стандартный фильтр из ткани «Петрянова» АФА-РМП-20. Измерение проводят с помощью дозиметра-радиометра МКС-1117М с альфа-датчиком. Отбирают две пробы объемом 1 и $10 \mathrm{~m}^{3}$. Сразу после отбора начинают измерение на фильтрах с интервалом 5 мин.

На рис. 2 приведены данные эксперимента по измерению $\alpha$-активности фоновых проб аэрозолей различного объема, полученные по методике [27] и показывающие динамику их распада. Видно, что временной промежуток, за который активность смеси радионуклидов в пробе уменьшается в 2 раза, составляет около 50 мин. Произведем расчет активности дочерних радионуклидов для каждой эманации через 1 ч, приняв начальное количество материнского радионуклида за 100 Бк, и оценим динамику распада по следующей формуле [28]:

$$
A_{i}(t)=A_{1}(0) \lambda_{1} \lambda_{2} \ldots \lambda_{i}\left[e^{\frac{-\lambda_{1} t}{\left(\lambda_{2}-\lambda_{1}\right)\left(\lambda_{3}-\lambda_{1}\right) \ldots\left(\lambda_{i}-\lambda_{1}\right)}}+\ldots+e^{\frac{-\lambda_{i} t}{\left(\lambda_{1}-\lambda_{i}\right)\left(\lambda_{2}-\lambda_{i}\right) \ldots\left(\lambda_{i-1}-\lambda_{i}\right)}}\right]
$$

где $A_{i}$ - активность дочернего радионуклида от материнского $A_{1}(0)$ через время $t$, Бк; $\lambda$ постоянная распада $=0,693 / T_{1 / 2}, T_{1 / 2}-$ период полураспада. В табл. 2 приведены данные расчетов.

Поскольку эманации представляют собой инертные газы, они на фильтры не осаждаются и в расчетах участия не принимают. Из полученных данных видно, что после часовой выдержки в пробе в значимых количествах должен присутствовать только свинец-214, также за один час $\alpha$-активность пробы должна уменьшиться примерно в 4 раза.

В результате сравнения данных, полученных экспериментально, с расчетными можно говорить о том, что они удовлетворительно согласуются с погрешностью измерения $30 \%$. Вместе с тем динамика распада смеси радионуклидов в эксперименте несколько ниже, чем расчетная.

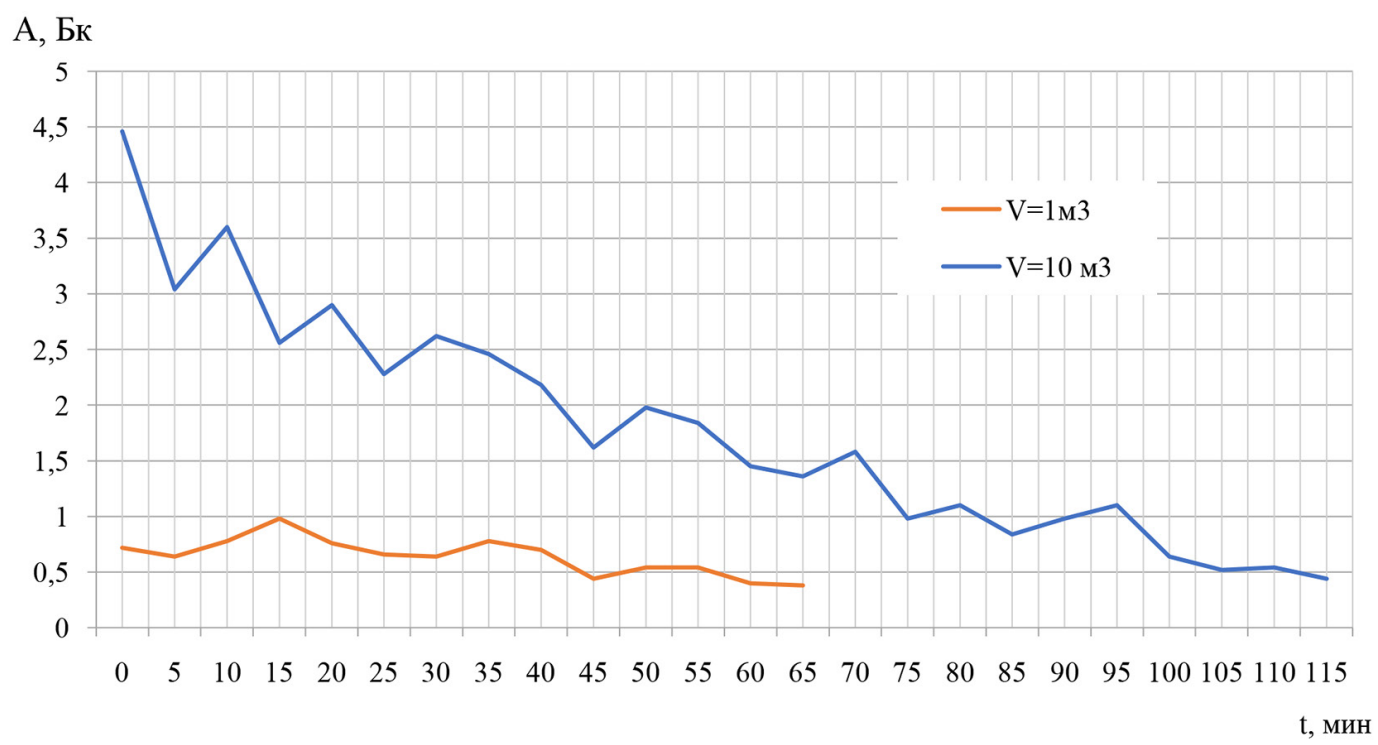

Рис. 2. Динамика распада естественных $\alpha$-активных радионуклидов в пробах аэрозолей

Fig. 2. The dynamics of decay of natural $\alpha$-active radionuclides in aerosol samples

$$
-971-
$$


Таблица 2. Активность дочерних радионуклидов от материнского активностью 100 Бк после часовой выдержки, Бк

Table 2. Activity of daughter radionuclides from maternal activity of $100 \mathrm{~Bq}$ after 1 hour exposure, Bq

\begin{tabular}{|c|c|c|c|c|c|}
\hline \multirow{2}{*}{ Эманация } & \multicolumn{2}{|c|}{ Материнский радионуклид } & \multicolumn{3}{|c|}{ Дочерние радионуклиды } \\
\cline { 1 - 6 } & $t_{0}$ & $t_{1 ч}$ & \multicolumn{3}{|c|}{$t_{14}$} \\
\hline \multirow{2}{*}{${ }^{222} \mathrm{Rn}$} & ${ }^{218} \mathrm{Po}$ & ${ }^{218} \mathrm{Po}$ & ${ }^{214} \mathrm{~Pb}$ & ${ }^{210} \mathrm{~Pb}$ & ${ }^{210} \mathrm{Po}$ \\
\cline { 2 - 6 } & 100 & 0,0002 & 26,4 & $1,50 \mathrm{E}-03$ & $2,30 \mathrm{E}-18$ \\
\hline \multirow{2}{*}{${ }^{220} \mathrm{Rn}$} & ${ }^{216} \mathrm{Po}$ & ${ }^{216} \mathrm{Po}$ & ${ }^{212} \mathrm{~Pb}$ & ${ }^{212} \mathrm{Po}$ & - \\
\hline & 100 & 0 & $3,99 \mathrm{E}-07$ & $3,32 \mathrm{E}-06$ & - \\
\hline \multirow{2}{*}{${ }^{219} \mathrm{Rn}$} & ${ }^{215} \mathrm{Po}$ & ${ }^{215} \mathrm{Po}$ & ${ }^{211} \mathrm{~Pb}$ & ${ }^{207} \mathrm{Tl}$ & - \\
\cline { 2 - 6 } & 100 & 0 & $3,99 \mathrm{E}-07$ & 1,06 & - \\
\hline
\end{tabular}

Возможно, это связано с влиянием прочих естественных радионуклидов, не задействованных в расчете.

Далее, с помощью той же формулы рассчитаем содержание свинца-214 в пробе через каждые 5 мин, таким образом практически симулируя эксперимент. Данные расчета приведены на диаграмме в сравнении с экспериментальными данными (рис. 3).

Из полученных зависимостей видно, что расчетная модель хорошо соотносится с экспериментальными данными. Таким образом, любое отклонение кривой экспериментальных данных вверх от расчетной модели должно расцениваться как обусловленное наличием техногенных радионуклидов в пробе. И такое отклонение, как видно из диаграммы,

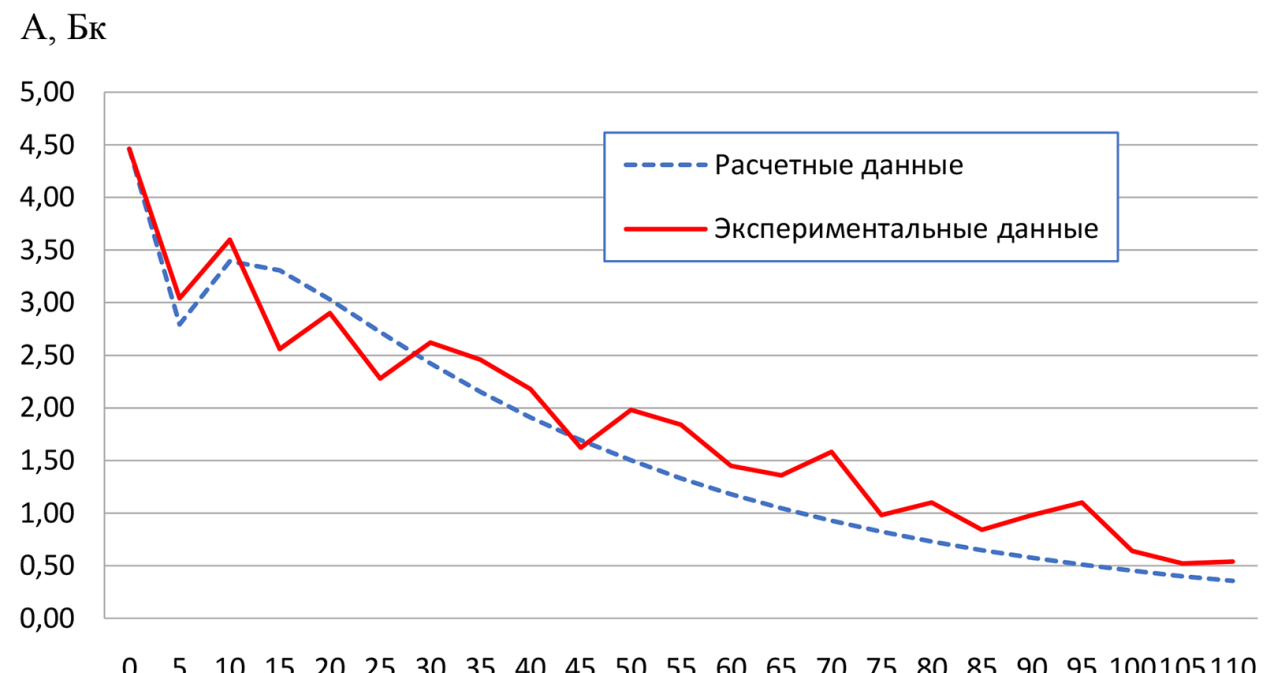

$t, \mathrm{Mин}$

Рис. 3. Экспериментальные данные распада смеси естественных радионуклидов в сравнении с расчетной моделью

Fig. 3. Experimental data on the decay of a mixture of natural radionuclides in comparison with the calculated model 
можно зафиксировать уже после получасовой выдержки. При этом само измерение не столь важно, необходимо фиксировать динамику распада в первые часы после отбора и четко фиксировать отклонения от калибровочной кривой. Сделать это представляется возможным без конструктивного изменения оборудования. Изменениям подвергнется лишь алгоритм измерений.

\section{Выводы}

Необходимо и возможно создание расчетной модели, сравнивающей динамику $\alpha$-распада пробы с некой калибровочной кривой распада, полученной для данного региона. И тогда можно уже через полчаса сделать вывод о наличии и конкретном количестве техногенных радиоактивных аэрозолей в воздухе помещений, тем самым сократив время определения этого в 72 раза.

\section{Благодарности / Acknowledgments}

Исследование выполнено при финансовой поддержке РФФИ и Правительства Красноярского края в рамках научного проекта № 18-48-242001 «Теплофизические и гидродинамические особенности кинетики смесеобразования при иммобилизации радиоактивных отходов в цементную матрицу с использованием эффектов кавитации».

The reported study was funded by RFBR and the government of Krasnoyarsk region according to the research projects no. 18-48-242001 Thermo physical and hydrodynamic features of the kinetics of mixture formation upon immobilization of radioactive waste in cement matrix using the effects of cavitation.

\section{Список литературы / References}

[1] Объединенная конвенция о безопасности обращения с отработавщим топливом и безопасности обращения с радиоактивными отходами. Вена, 5 сентября 1997 года. Конвенция вступила в силу для России 19.04.2006. http://www.bellona.ru/Casefiles/vienna97 [Joint Convention on the Safety of Spent Fuel Management and the Safety of Radioactive Waste Management. Vienna, 5th September 1997. The Convention entered into force for Russia on 19.04.2006. http://www. bellona.ru/Casefiles/vienna97 (in Russian)].

[2] Конвенция о ядерной безопасности. Вена, 17 июня 1994 года. Конвенция вступила в силу для России 24.10.1996. http://www.bellona.ru/Casefiles-/vienna94 [Convention on Nuclear Safety. Vienna, 17th June 1994. The Convention entered into force for Russia on 24.10.1996. http:// www.bellona.ru/Casefiles-/vienna94 (in Russian)].

[3] Безопасность ядерного топливного ичилла; пер. с англ. М.: ЦНИИАТОМИНФОРМ, 2002. 196 c. [The Safety of the Nuclear Fuel Cycle (in Russian)].

[4] Шведов В.П., Седов В.М., Рыбальченко И.Л., Власов И.Н. Ядерная технология. М.: Атомиздат, 1979. 536 с. [Shvedov V.P., Sedov V.M., Ribalchenko I.L., Vlasov I.N. Nuclear technology. Moscow: Atomizdat, 1979. 536 p. (in Russian)].

[5] Публикаџия 103 Международной Комиссии по радиащионной защите (МКР3); пер с англ., ред. М.Ф. Киселев и Н.К. Шандала. М.: Изд. ООО ПКФ «Алана», 2009. 344 с. [International Commission on Radiation Protection Publication 103 (MKRZ); translated, edit. M.F. Kiselev and N.K. Shandala. Moscow: Publishing House. OOO PKF «Alana», 2009. 344 p. (in Russian)].

$$
-973-
$$


[6] Ключников А.А., Пазухин Э.М., Шигера Ю.М., Шигера В.Ю. Радиоактивные отходы АЭС и методы обрашения с ними. Киев: Институт проблем безопасности АЭС НАН Украины, 2005. 487 с. [Kluychnikov A.A., Pazukhin A.M., Shigera U.M., Shigera V.U. Nuclear power plant radioactive waste and methods of handling them. Kiev: Institute for NPP Safety Problems, National Academy of Sciences of Ukraine, 2005. 487 p. (in Russian)].

[7] Кузнецов В.М. Основные проблемы и современное состояние предприятий ядерного топливного цикла Российской Федерации. М.: Российская Демократическая партия «Яблоко», 2002. 259 c. [Kuznetsov V.M. The main problems and the current state of the nuclear fuel cycle of the Russian Federation. Moscow: Russian Democratic Party "Yabloko", 2002. 259 p. (in Russian)].

[8] Лебедев В.М. Ядерный топливный изикл. М.: Энергоатомиздат, 2005. 305 с. [Lebedev V.M. Nuclear fuel cycle. Moscow: Energoatomizdat, 2005. 305 p. (in Russian)].

[9] Федеральный закон от 21.11.95 N 170-Ф3 (ред. от 02.07.2013 с изменениями, вступившими в силу 02.09.2013) «Об использовании атомной энергии» [The Federal Law 21.11.95 N 170-FZ (edit. 02.07.2013 with changes, to take effect 02.09.2013) «On nuclear energy use» (in Russian)].

[10] Безопасность России. Правовые, социально-экономические и научно-технические аспекты. Регулирование ядерной и радиаџионной безопасности / Колл. авт. М.: МГОФ «Знание», НТЦ ЯРБ, 2003. 400 с. [Security of Russia. Legal, socio-economic and scientific and technical aspects. Regulation of nuclear and radiation safety / team of authors Moscow: MGOF «Znanie», 2003. 400 p. (in Russian)].

[11] Санитарные правила обращения с радиоактивными отходами (СПОРО-2002). СП 2.6.6.1168-02. СПб.: Деан, 2003. 64 с. [Sanitary rules for radioactive waste management (SPOR2002). SP 2.6.6.1168-02. Spb, Dean, 2003. 64 p. (in Russian)].

[12] Нормы радиационной безопасности (НРБ-99/2009): Санитарно-эпидемиологические правила и нормы. М.: Федеральный центр гигиены и эпидемиологии Роспотребнадзора, 2009. 100 c. [Radiation Safety Standards (NRB-99/2009): Sanitary and Epidemiological Rules and Norms. Moscow: Federal Center for Hygiene and Epidemiology of Rospotrebnadzor, 2009. 100 p. (in Russian)].

[13] Основные санитарные правила обеспечения радиаџионной безопасности (ОСПОРБ 99/2010): Санитарные правила и нормативы. М.: Федеральный центр гигиены и эпидемиологии Роспотребнадзора, 2010. 83 с. [Basic sanitary rules for ensuring radiation safety (OSORB 99/2010): Sanitary rules and regulations. Moscow: Federal Center for Hygiene and Epidemiology of Rospotrebnadzor, 2010. 83 p. (in Russian)].

[14] Волков В.Г., Чесноков А.С. Радиоактивные отходы: хранение и переработка. Промышленные ведомости. 2011, 11-12. [Volkov V.G., Ghesnokov A.S. Radioactive waste: storage and processing. Industrial statements. 2011, 11-12 (in Russian)].

[15] Милютин В.В., Гелис В.М. Современные методы очистки жидких радиоактивных отходов и радиоактивно-загрязненных природных вод. М.: ИФХЭ РАН, 2011. [Miluytin V.V., Gelis V.M. Modern methods of purification of liquid radioactive waste and radioactively contaminated natural waters. Moscow: IFHE RAS, 2011 (in Russian)].

[16] Кулагина Т.А., Кулагин В.А., Матюшенко А.И. Техносферная безопасность в ядерной энергетике. Красноярск: Гротеск, СФУ, 2014. 286 с. [Kulagina T.A., Kulagin V.A., Matushenko A.I. Technosphere safety in nuclear power Krasnoyarsk: Grotesk, SibFU, 2014. 286 p. (in Russian)].

$$
-974-
$$


[17] Кулагина Т.А., Козин О.А., Матюшенко А.И. Экологическая безопасность техносферных объектов. Красноярск: Гротеск, 2015. 323 с. [Kulagina T.A., Kozin O.A., Matushenko A.I. Environmental safety of technosphere objects. Krasnoyarsk: Grotesk, 2015. 323 p. (in Russian)].

[18] Кулагин В.А., Кулагина Т.А., Матюшенко А.И. Переработка отработавшего ядерного топлива и обращение с радиоактивными отходами. Журн. Сиб. федер. ун-та. Техника и технологии. 2013. 6(2). 123-149. [Kulagin V.A., Kulagina T.A., Matushenko A.I. Reprocessing of spent nuclear fuel and radioactive waste management. Journal of Siberian Federal University. Engineering and Technologies. 2013, 6(2), 123-149 (in Russian)].

[19] Кулагина Т.А., Кулагин В.А., Москвичев В.В., Попков В.А. Применение кавитационной технологии в процессах обращения с отработавшим ядерным топливом, Экология и промылиленность России, 2016, 20(10), 4-10. DOI: 10.18412/1816-0395-2016-10-4-10 [Kulagina T.A., Kulagin V.A., Moskvichev V.V., Popkov V.A. Application cavitation technology in the treatment of spent nuclear fuel processes, Ecology and industry of Russia, 2016, 20(10), 4-10. DOI: 10.18412/18160395-2016-10-4-10 (in Russian)].

[20] Кулагина Т.А., Кулагин В.А. Растворение алюмосиликатных осадков в хранилищах жидких РАО. Радиоактивные отходы. 2020. 2(11). 75-84. DOI: 10 25283/2587-9707-2020-2-7584 [Kulagina T.A., Kulagin V.A. Aluminosilicate Sediment Dissolution in Liquid Radioactive Waste Storage Facilities. Radioactive waste, 2020, no. 2(11), 75-84. DOI: 10.25283/2587-9707-2020-2-75-84 (in Russian)].

[21] Теверовский Е.Н., Артемова Н.Е., Бондарев А.А. и др. Допустимые выбросы радиоактивных и химических веществ в атмосферу; ред. Е.Н. Теверовский и И.А. Терновский. 2-е изд., перераб. и доп. М.: Энергоатомиздат, 1985. [Teverovskiy Y.N., Artemova N.Y., Bondarev A.A. etc. Permissible emissions of radioactive and chemical substances into the atmosphere; edit. Y.N. Teverovskiy and I.A. Ternovskiy. 2nd edition, revised and enlarged. Moscow: Energoatomizdat, 1985. (in Russian)].

[22] Еремеев И.С. Автоматизированные системы радиаџионного мониторинга окружающей среды; отв. ред. А.И. Кондалев. Киев: Наукова Думка, 1990. [Eremeev I.S. Automated systems for radiation monitoring of the environment; editor-in-chief A.I. Kondalev. Kiev: Naukova Dumka, 1990 (in Russian)].

[23] Жабо В.В. Охрана окружающей среды на ТЭС и АЭС. М.: Энергоатомиздат, 1992. [Jhabo V.V. Environmental protection at thermal power plants and nuclear power plants. Moscow: Energoatomizdat, 1992 (in Russian)].

[24] Эйзенбад М. Радиоактивность внешней среды; пер. с англ. под ред. П.П. Лярского. М.: Атомиздат, 1967. [Ayzenbad M. Environmental radioactivity; translated from English P.P. Lyarskiy. Moscow: Atomizdat, 1967. (in Russian)].

[25] Голубев Б.П. Дозиметрия и защзита от ионизирующих излучений. Изд. 3-е, перераб. и доп. под ред. Е.Л. Столяровой. М.: Атомиздат, 1976. [Golubev B.P. Dosimetry and protection against ionizing radiation. Ed. 3rd, rev. and add. edited by. E.L. Stolyarova. Moscow: Atomizdat, 1976 (in Russian)].

[26] Перцов Л.А. Природная радиоактивность биосферыл. М.: Атомиздат, 1964. 311 с. [Pertsov L.A. Natural radioactivity of the biosphere. Moscow: Atomizdat, 1964. 311 p. (in Russian)].

$$
-975-
$$


[27] Методика выполнения измерений. Обьемная активность радиоактивных газов и аэрозолей (МВИ-РЗП-ГХК-2016) МВИ 01-13.021-2016: Железногорск. 2016. 43 с. [Measurement technique. Volume activity of radioactive gases and aerosols (MVI-RZP-GHK-2016) MVI 01-13.021-2016: Zheleznogorsk. 2016. 43 p. (in Russian)].

[28] Гусев Н.Г., Дмитриев П.П. Радиоактивные цепочки: Справочник. 2-е изд., перераб. и доп. М.: Энергоатомиздат, 1988. 112 с. ISBN 5-283-02969-7. [Gusev N.G., Dmitriev P.P. Radioactive Chains: A Handbook. 2nd ed., Rev. and additional. Moscow: Energoatomizdat, 1988. 112 p. ISBN 5-283-02969-7 (in Russian)]. 